\title{
WILEY-VCH
}

\section{An Assistive Magnetic Skin System: Enabling Technology for Quadriplegics}

Abdullah S. Almansouri ${ }^{*}$,Lakshmeesha Upadhyaya, Suzana P. Nunes, Khaled N. Salama, and Jurgen Kosel ${ }^{*}$

A. S. Almansouri, Prof. K. N. Salama and Prof. J. Kosel

Computer, Electrical, and Mathematical Sciences and Engineering Division (CEMSE) King Abdullah University of Science and Technology (KAUST)

Thuwal 23955-6900, Saudi Arabia

E-mail: abdullah.almansouri@kaust.edu.sa

jurgen.kosel@kaust.edu.sa

A. S. Almansouri

Department of Electrical and Electronic Engineering, University of Jeddah, Dhahban 23881, Saudi Arabia.

Prof. J. Kosel

Sensor Systems Division, Silicon Austria Labs

High Tech Campus Villach

Europastraße 12, A-9524 Villach, Austria

K. N. Salama

Sensors Lab, Advanced Membranes \& Porous Materials Center (AMPMC), Computer Electrical Mathematical Science and Engineering (CEMSE) Division, King Abdullah University of Science and Technology (KAUST), Thuwal, 23955-6900, Saudi Arabia

Lakshmeesha Upadhyaya and Prof. Suzana P. Nunes

Biological and Environmental Science and Engineering Division (BESE), Advanced Membranes and Porous Materials Center (AMPM), Thuwal, 23955-6900, Saudi Arabia.

Keywords: wearable electronics, flexible magnet, magnetic skin, assistive technology, quadriplegic 


\title{
WILEY-VCH
}

\begin{abstract}
People with quadriplegia no longer have control over their legs, neither the hands and cannot continue living their life independently. On top of that, severely injured quadriplegics (i.e., $\mathrm{C} 1$ and $\mathrm{C} 2$ injuries) suffer from speaking difficulties and minimal head and neck movements. With the advancement in wearable artificial skins and the Internet of Things, realizing comfortable and practical solutions for quadriplegics is more tangible than ever. Here, a comprehensive assistive magnetic skin system is presented that allows quadriplegics, including the severely injured ones, to move around individually and control their surroundings with ease. The system tracks facial expressions by tracking the movement of magnetic tattoos attached to the face, using magnetic field sensors incorporated into eyeglasses. The magnetic tattoos are made of highly flexible, stretchable, breathable, and biocompatible magnetic skins. In combination with smart-glasses, smart-wheelchair, and smart-gadgets, the users can move around and control their environment with their facial expressions. The system is also designed to allow quadriplegics to perform outdoor activities effortlessly. It supports line-of-sight communication and does not require pretethering to the smart-gadgets, unlike the existing solutions. Thus, enabling the user to walk on pathways, activate pedestrian lights, control public elevators, and perform various outdoor activities independently.
\end{abstract}




\section{WILEY-VCH}

\section{Introduction}

Every year, about 17,730 people in the United States only, incur spinal cord injuries (SCI). In other words, about 48 people every day lose some functions of their bodies, due to traumatic damage in the spinal cord. In many cases, these injuries are severe, making it challenging for the injured person to live independently for the rest of their lives. ${ }^{[1]}$ Assistive technologies are used by injured people (e.g., paraplegia), allowing them to commute more independently. Yet, people with severe injuries (e.g., quadriplegia-paralyzed) cannot use their hands and legs, making it impossible to use conventional assistive technologies (i.e., powered-wheelchairs using a joystick) for moving around. ${ }^{[2,3]}$ Nevertheless, SCI mostly occurs to the middle-aged adults (around 43 years old); hence, they need long-term solutions for improving their life experience, providing them with the ability to move around and control their environment independently. ${ }^{[1]}$

Existing assistive solutions for people with quadriplegia include spin-n-puff, head or chin joysticks $;{ }^{[4]}$ brain-machine interfaces using neural-detectors to interpret action commands; ${ }^{[5,6]}$ camera-based systems for facial and gaze detection; ${ }^{[7,8]}$ voice detection; and tongue detection. ${ }^{[9]}$ However, these technologies offer limited action commands. They require specific, continuous, and sophisticated setups that are not suitable for all types of patients. Continuous attention by the patient is needed (i.e., prevent the user from moving and talking at the same time). The set-ups are bulky and disturb the patients (e.g., bulky transducers attached to sensitive organs), are expensive, or require significant computational power. ${ }^{[4-10]}$

Sever quadriplegics with $\mathrm{C} 1$ and $\mathrm{C} 2$ injuries suffer from speaking difficulties and very limited neck and head movements. ${ }^{[1]}$ As a consequence, the only remaining solutions for them are either cameras, tongue control, or neural detectors. ${ }^{[9,12]}$ Nevertheless, these technologies, so far, have shown very limited action commands. ${ }^{[9,12]}$ They can only offer limited controlling abilities 


\section{WILEY-VCH}

(i.e., only moving a wheelchair) ${ }^{[9]}$, yet, not sufficient for moving around and controlling the environment independently. In other words, it is challenging to find a comprehensive solution for the severely injured quadriplegics that helps them to live their life independently. Hence, there is a need for more comfortable, efficient, and functional solutions to facilitate the daily life of quadriplegics including the severely injured ones. With the current advancements in artificial skins and the Internet-of-Things (IoT), such a solution is more viable than ever. The unique advantage of artificial skins is the ability to hide their presence, visually and perceptively, making them comfortable for everyday use.

In this work, an assistive magnetic skin system (AM2S) for severe quadriplegics is implemented using a breathable, biocompatible, and extremely flexible magnetic skin. The AM2S offers a new technique for tracking facial expressions. Combined with a dedicated line-of-sight communication protocol, it allows the user to move around independently and control the surrounding gadgets with ease.

\section{Concept}

The AM2S is envisioned as a unified solution that allows quadriplegics to have full control of their surroundings. The AM2S consists of: a) magnetic skins (i.e., a biocompatible, highly flexible and stretchable magnetic composite) attached to different locations on the face (e.g., forehead and nose), b) smart-glasses with integrated magnetic field sensors and Bluetooth connectivity, c) a smart-wheelchair with Bluetooth and infrared (IR) connectivity, and d) smartgadgets that support IR connectivity. Figure 1 shows an illustration of a quadriplegic using the AM2S for independent movement and independent control of the surroundings (e.g., lights, elevator, curtains, television, computer, etc.). The AM2S is designed to allow the user, for instance, 


\section{WILEY-VCH}

to open gates, perform outdoor walk, activate pedestrian lights, control elevators, and perform the desired tasks effortlessly and independently.

The correct placement of the magnetic skin is on the face. This is because the facial muscles, jaws, and eyes are stimulated by the cranial motor nerves. These cranial nerves are protected by the skull; hence, they are less likely to get injured during accidents. ${ }^{[11,13,14]}$ In other words, severely injured people are more likely to keep full control over their facial muscles. The operation of the AM2S is as follows: the smart-glasses, in combination with the magnetic skin, track the facial expressions of the user. These expressions are then translated into movement commands to move the wheelchair or control commands to control the surrounding environment of the user. The controlling commands are carried-on to the smart-gadgets using IR communications. The IR communication allows the user to control any smart-gadget of interest by aiming at that device. This is because the IR communication is directive (i.e., it uses line-of-sight communication), and universal (i.e., seamless connectivity). For instance, for the case of controlling the lights, the user aims at the light-switch and uses the corresponding facial gestures (e.g., eyebrow-up) to turn the lights on. Supplementary Movie 1 shows a demonstration of the AM2S, where the user moves around and controls the lights using facial expressions.

\section{Implementation and Considerations}

\subsection{Placement of the Magnetic Skin}

The exact position of the magnetic skin can be altered to different locations on the face to suit the specific needs of any user. Furthermore, humans commonly express their feelings and emotions with facial expressions. Therefore, the location of the magnetic skin should be chosen in a way to avoid accidental activations, yet, to allow triggering of intended activations. For example, 


\section{WILEY-VCH}

cheeks are easily controlled; however, they also move when talking and laughing. As a consequence, having a magnetic skin attached to the cheek is not practical and results in a lot of accidental activations. On the other hand, moving one eyebrow up while the other eyebrow is stationary is less likely to occur accidentally, yet, not everyone can perform this expression with ease. Based on trials and the users' feedback, the implemented locations of the magnetic skin are on the forehead (e.g., hidden in an eyebrow or placed on the glabella), and on the nose (left and right sides). For such configuration, the user has facial control by moving the eyebrows up and down and tilting the nose left and right. These gestures are practical, comfortable, and easy to learn, but are meant here only as examples and can be adjusted to other preferences and needs in many ways. In fact, the system was tested on three different people, and they independently managed to use it within a few minutes of training. Furthermore, the users managed to hold long conversations without accidentally activating the wheelchair, evidencing the practicality of this setup.

\subsection{The Smart-glasses}

The smart-glasses mainly consist of four-magnetic field sensors, a microcontroller, a Bluetooth module, and a battery, as shown in Figure 2a. Three magnetic field sensors are located in proximity to the magnetic skins (e.g., two at the nose and one at the glabella). The fourth magnetic field sensor is placed far away from the magnetic skins and acts as a reference sensor. This reference sensor is used for the cancelation of external magnetic fields (i.e., Earth's magnetic field). In other words, all the measurements are differential, hence, increasing the robustness of the readings. The complete design of the smart-glasses is discussed in Supplementary Information 1. Note that while our prototype implementation utilizes a generic microcontroller platform, all components could be neatly integrated into the frame of the glasses, ensuring 


\section{WILEY-VCH}

comfortable and unobtrusive wear. In this configuration, the magnetic skins are always placed above or below the magnetic field sensors, and the facial expressions are translated into a change of the distances between the magnetic skins and the magnetic field sensors along the Z-axis. Thus, the magnetic skin has been magnetized along the Z-axis, and the magnetic field sensors are sensitive to the Z-axis only. In other words, the facial expressions are translated into changes of the magnetic field along the Z-axis. With this setup, we implemented thirteen different gestures based on the combinations of the nose and the eyebrows movements. These gestures include noexpression, eyebrow-up, eyebrow-down, nose-right, and noise-left, as shown in Figure 2b. More gestures can be added; for instance, double eyebrow-up, triple eyebrow-up, double eyebrow-up and nose-right, and double eyebrow-up and nose-left, etc. The complete list of the gestures and examples of their applications are presented in Supplementary Information 2.

\subsection{The Smart-wheelchair}

The smart-wheelchair is a motorized wheelchair combined with a microcontroller, a Bluetooth module for wireless communication with the smart-glasses, and an IR transmitter/receiver for the wireless connection with the smart-gadgets, as shown in Figure 2c. The head-unit acts as the central hub for the AM2S that receives and processes the intended commands from the smart-glasses, controls the motors of the wheelchair, and handles the communications with the smart gadgets. The complete design of the smart-wheelchair, including the head-unit, is discussed in Supplementary Information 3. 


\section{WILEY-VCH}

\subsection{The AM2S system}

The combination of the smart-glasses, the smart-wheelchair, and the smart-gadgets is an enabling technology for quadriplegics. Figure 2d shows the flow chart of the AM2S. The smartglasses track the movement of the magnetic skin, once the movement passes a predefined threshold, this movement is translated into one of the thirteen gestures (processing methods and digital representation of the facial expressions are discussed in Supplementary Information 2). These gestures are then transmitted to the head-unit using Bluetooth communication. The headunit then determines the type of the action commands (i.e., moving commands or controlling commands). For the case of the moving commands, the head-unit sends the commands to the wheelchair to move according to the gestures (i.e., the facial expression). As a consequence, the chair moves forward, moves backward, turns right, or turns left. For the case of the controlling commands, the head-unit sends the commands to the IR link to control the smart-gadgets within the line-of-sight. These commands are further processed at the smart-gadgets for the intended use, which can be different from one smart-gadget to another. For example, an eyebrow-up command can turn on the light, and the same gesture can be used to move the cursor up on a computer. Note, the triple eyebrow-up command is used as a search/disable command, and it is reserved for switching between the moving commands and the controlling commands (Supplementary

\section{Information 4).}

Table 1 presents the training time and the success rate of the AM2S in recognizing the facial expressions (i.e., eyebrows-up, eyebrows-down, nose-right, and nose-left gestures) for three different users. The table also highlights the total number of unintentional activations that occurred during the success rate test or during a two-minute session of free talk (i.e., the user is allowed to move freely, laugh, etc.). It requires less than 15 minutes of training to operate the system 


\section{WILEY-VCH}

independently and with a high confidence level. Furthermore, the average success rate exceeds $93 \%$ for the worst-case scenario, with a minimum average accidental activation of about 0.3 activations during the full testing session. Furthermore, the users did not complain about any sort of fatigue while operating the system during the full period of training and testing period. Note, this project is approved and regulated by the Institutional Biosafety and Bioethics Committee (IBEC) of King Abdullah University of Science and Technology (IBEC number 18IBEC19), and supported by informed written consent by all participants.

Table 2 compares the AM2S solution with the most popular assistive technologies for quadriplegics. Unlike the existing solutions, the AM2S is comprehensive, comfortable to use, compatible for indoor and outdoor applications, and supports severe quadriplegic injuries. On top of that, the AM2S supports line-of-sight communication to directly control any compatible device without the need for a pre-tethering. Thus, making it the only truly outdoor-ready solution that allows the user to directly control any smart-gadget within reach.

\section{Realization of breathable and stretchable magnetic skins}

The magnetic skin is stretchable, flexible, comfortable, and biocompatible. ${ }^{[15]}$ Figure 3 shows a photograph of a user wearing the smart-glasses and three patches of the magnetic skin (i.e., one on the glabella and two on the nose). As presented, the magnetic skin can be realized in any shape; for example, on the glabella, it is shaped like the word "ENABLE," thus, allowing for customizable personal preferences. The magnetic skin is realized by mixing a silicon-based elastomer matrix (Ecoflex, Smooth-on) with a permanent magnetic powder (NdFeB) with a 1:1 weight ratio. This ratio offers the best combination of the high remanent magnetization and high flexibility (Supplementary Information 5) ${ }^{[15]}$ For such condition, a $1: 1 \mathrm{wt} \% 10 \times 2 \times 0.7 \mathrm{~mm}^{3}$ magnetic skin sample has a magnetic flux density of $177 \mu \mathrm{T}$ at $7 \mathrm{~mm}$ distance. Such a high 


\section{WILEY-VCH}

magnetic field is sufficient to be detected by the magnetic field sensors with good signal to noise ratio. The magnetic skin is durable and these enhanced properties is maintained over 1000 stress cycles. ${ }^{[15]}$ Furthermore, the magnetic skin biocompatibility is evidenced by the results of a PrestoBlue cell viability test, where cells maintain a high viability (i.e., $>90 \%$ ), when cultured on top of a magnetic skin for three days. ${ }^{[15]}$

Due to the extended duration of wearing the magnetic skin, breathability becomes critical. In fact, according to Miyamoto et al. ${ }^{[16]}$, breathable artificial skins can suppress irritations and other feelings of discomfort that might arise from wearing artificial skins. The breathability of the magnetic skin is attained by having micro-holes structured in the skin. Figure 4a-d shows the fabrication steps of the breathable magnetic skin. After preparing the magnetic skin sample, microholes are machined using a $30 \mathrm{~W}$ ytterbium fiber laser with a $1.06 \mu \mathrm{m}$ wavelength. The breathable magnetic skin offers a hole-density of up to 2500 holes $/ \mathrm{cm}^{2}$ (e.g., Figure 4f), which is more than 4-times the density of the humans' sweat glands. ${ }^{[17]}$ Both the size and the density of the holes can be adjusted as needed. Figure 4e shows an optical image of a diluted magnetic skin sample highlighting the uniform distribution of the magnetic particles. Figure 4f-g show the breathable magnetic skin with hole densities of 1250 holes $/ \mathrm{cm}^{2}$ and 2500 holes $/ \mathrm{cm}^{2}$. The micro-holes shown in the SEM image in Figure 4h have a diameter of about $95 \mu \mathrm{m}$. Figure 4i presents the stressstrain curves of the magnetic skin as a function of the hole density. As the hole density increases, the Young's modulus decreases, and thus the material becomes more elastic. The measured Young's modulus is $134 \mathrm{kPa}, 67 \mathrm{kPa}$, and $50 \mathrm{kPa}$ for the no-holes, 1250 holes $/ \mathrm{cm}^{2}$, and 2500 holes $/ \mathrm{cm}^{2}$ samples, respectively. In other words, the breathable magnetic skin is twice more elastic than a plain magnetic skin and more than 32 times more elastic than the conventional Sylgardbased magnetic composite with Young's modulus greater than $2200 \mathrm{kPa} \cdot{ }^{[15,18,19]}$ Note that, despite 


\section{WILEY-VCH}

the presence of the holes, the breathable magnetic skin is durable and can withstand more than $400 \%$ elongation without breaking. Figure $\mathbf{4} \mathbf{j}$ shows the magnetization curve of the composite. It offers a high remanent magnetization (i.e., the magnetic field embedded in the magnetic skin after being magnetized) of $126 \mathrm{mT}$, and a high coercivity (i.e., the needed external field to demagnetize the magnetic skin) of $0.56 \mathrm{~T}$.

Quantitively, breathability is characterized by the water vapor transmission rate (WVTR), which is a measure of the vapor permeability of a substrate. The WVTR of the human skin is in the range of $200-500 \mathrm{~g} \cdot \mathrm{m}^{-2}$. day ${ }^{-1}{ }^{[20]}$ The magnetic skin is highly breathable and offers a WVTR of up to $95 \times 10^{3}$ g.m ${ }^{-2}$.day ${ }^{-1}$ (Figure $4 \mathbf{k}$ ), which is about 2 orders of magnitude higher than the one of human skin. Note, the holes density has a positive impact on the breathability (breathability increases with holes density) and a negative impact on the magnetic field strength (magnetic field decreases by increasing holes density). The measurement results show that the magnetic field drops by about $20 \%$ for every added 1250 holes per $\mathrm{cm}^{2}$ (about $1 \%$ per every 62 holes). Thus, a sensible hole density is 1250 holes $/ \mathrm{cm}^{2}$ as it offers high breathability of $60 \times 10^{3} \mathrm{~g} \cdot \mathrm{m}^{-2}$.day ${ }^{-1}$ while reducing the magnetic field by only $20 \%$.

\section{Conclusion}

In summary, the AM2S utilizes breathable magnetic skins to provide a comprehensive assistive solution for quadriplegics. The AM2S is realized by attaching magnetic skins to different places on the face that remain functional even when undergoing sever $\mathrm{C} 1$ and $\mathrm{C} 2$ injuries. This skin is highly breathable with a WVTR of up to $95 \times 10^{3}$ g.m $\mathrm{m}^{-2}$.day ${ }^{-1}$, allowing for comfortable extended wear. In combination with smart-glasses that integrate magnetic field sensors, the AM2S tracks the magnetic skins, and correspondingly, the facial expressions of the user. The integration 


\section{WILEY-VCH}

of a smart-wheelchair and smart-gadgets with the AM2S enables quadriplegics to move around and control their environment with facial expressions. The AM2S system supports thirteen different gestures, thus, providing the user with many controlling commands as a comprehensive solution for controlling their environment.

\section{Methods}

Smart-glasses design. The smart-glasses were manufactured using a printed circuit board (PCB) shaped as the frame of the glasses. It consists of four magnetic field sensors (BM1422AGMV) connected to one 8-channels multiplexer (PCA9548). A microcontroller with a built-in Bluetooth 4.0 module (Bluno nano, DFRobot) is powered by a $5 \mathrm{~V}$ battery and used to read and process the data from the magnetic field sensors. These electronics are communicating using Inter-Integrated Circuit $\left(\mathrm{I}^{2} \mathrm{C}\right)$ communication protocols with a $4.7 \mathrm{k} \Omega$ pull-up resistors.

Head-unit design. The head-unit PCB is realized using a microcontroller with a built-in Bluetooth 4.0 module (Bluno nano, DFRobot) and connected to two Digital-to-Analog converters (DACs, MCP4725), an IR transmitter and IR receiver operating at $38 \mathrm{kHz}$. The outputs of the two DACs are connected to the joystick port in the wheelchair, replacing and mimicking the output voltage of the original-joystick. For example, a high-voltage (e.g., $5 \mathrm{~V}$ ) of the DAC1 moves the wheelchair forward, and a low-voltage (e.g., $5 \mathrm{~V}$ ) of the DAC1 moves the wheelchair backward.

Smart-gadgets design. The smart-gadgets are realized using a microcontroller (Bluno nano, DFRobot), an IR transmitter and IR receiver operating at $38 \mathrm{kHz}$. The microcontroller is then interfaced with the gadget using direct connection, DACs, and/or relays, depending on the application. For example, for the case of using the AM2S for controlling a computer, the interface between the microcontroller and the computer is simply a USB cable. One the other hand, the interface could be a high-power relay for controlling a light-bulb. 


\section{WILEY-VCH}

Success rate test. The success rate test is achieved by doing the following. 1) introducing the system and the operating methodology to each user independently. 2) Each user gets a chance practicing using the system until they become comfortable using it. Once the user finish training, thy inform us and this time is considered as the training time. 3) The success test starts and the user is asked to perform the eyebrows-up, eyebrows-down, nose-right and nose-left gestures in random order and for a total of 10 times of each gesture. For each of these 40 activations (i.e., 10 activations per gesture), successfully activating the AM2S in the correct way is calculated as a success trial for the corresponding gesture. 4) The user stops making the gestures and is asked to talk for two minutes and to behave freely (i.e., move freely, laugh, etc.). Any accidental unintended activation of the system during the testing period or the free-talk period is counted as an accidental activation.

magnetic skin preparations. The magnetic skin is prepared by mixing EcoFlex 00-50 (Smooth-on) with micromagnetic powder (MQP-16-7FP NdFeB). The mixing ratio is 1:1 wt $\%$ (i.e., $25 \mathrm{wt} \%$ EcoFlex part A, $25 \mathrm{wt} \%$ EcoFlex part A and $50 \mathrm{wt} \% \mathrm{NdFeB}$ ). Then the mixture is vacuum desiccated for about 10 minutes to remove air bubbles. After that, the mixture is cast on a poly(methyl methacrylate) (PMMA) surface, planarized using a casting knife, and kept to dry at room temperature for 3 hours. Note that using a glass surface is not recommended, as the EcoFlex strongly adheres to it; hence, it is hard to detach the magnetic skin from the glass after curing. As an alternative approach, the magnetic skin can be molded into the desired shape and thickness. Regardless, after curing, the micro-holes are realized using a $30 \mathrm{~W}$ ytterbium fiber laser (Universal laser systems, PLS6MW) with a $1.06 \mu \mathrm{m}$ wavelength. Then the breathable magnetic skin is magnetized using a $1.8 \mathrm{~T}$ magnetic field. 


\section{WILEY-VCH}

Magnetic skin dimensions and attachments to the user. Tracking the facial expressions is achieved using $10 \times 2 \times 0.7 \mathrm{~mm}^{3}$ magnetic skin patches that are magnetized along the width (i.e., $2 \mathrm{~mm}$ side of the magnetic skin). The patches are attached to the skin of the user using a Vaseline ${ }^{\circledR}$ (a biocompatible and clinically proven petroleum jelly). Nevertheless, other biocompatible glue can also be used.

Water vapor transmission rate measurement. A mixed water vapor/gas test method was used to analyze the permeance of the magnetic skin. In this method, the sweep gas is maintained at one side of the skin (i.e., the permeate side) acting as a driving force for extracting the water vapor from the other side (i.e., the feed side). Humidity and temperature sensors were used to accurately measure these parameters on both sides of the skin. Then, the water vapor permeation through the magnetic skin is estimated using the following equation:

$$
Q_{\text {Vapor }}=Q_{N 2} * \gamma_{H 20} * V_{m} / M_{W, H 20}
$$

where $Q_{N 2}\left(\mathrm{~cm}^{3} \mathrm{~s}^{-1}\right)$ is the nitrogen flow rate at the permeate side, $\gamma_{H 20}\left(\mathrm{gm}^{-1}\right)$ is the absolute humidity, $V_{m}\left(\mathrm{~L} \mathrm{~mol}^{-1}\right)$ is the volume of $1 \mathrm{~mol}$ of penetrant at standard temperature and pressure and $M_{W, H 20}\left(\mathrm{gmol}^{-1}\right)$ is the molecular weight of water. 


\section{WILEY-VCH}

\section{Supporting Information}

Supporting Information is available from the Wiley Online Library or from the author.

\section{Conflict of Interest}

The authors declare no conflict of interest.

\section{Keywords}

Wearable electronics, flexible magnet, magnetic skin, assistive technology, quadriplegic

Received: ((will be filled in by the editorial staff))

Revised: ((will be filled in by the editorial staff))

Published online: ((will be filled in by the editorial staff)) 


\section{WILEY-VCH}

\section{References}

[1] NSCISC, Vol. 2020 (Ed: N. S. C. I. S. Center.), National Spinal Cord Injury Statistical Center, National Spinal Cord Injury Statistical Center. 2018.

[2] L. R. Hochberg, M. D. Serruya, G. M. Friehs, J. A. Mukand, M. Saleh, A. H. Caplan, A. Branner, D. Chen, R. D. Penn, J. P. Donoghue, Nature 2006, 442, 164.

[3] W. H. Organization, I. S. C. Society, International perspectives on spinal cord injury, World Health Organization, 2013.

[4] J. Leaman, H. M. La, IEEE Transactions on Human-Machine Systems 2017, 47, 486.

[5] L. R. Hochberg, J. P. Donoghue, IEEE Engineering in Medicine and Biology Magazine 2006, 25, 32.

[6] G. M. Paul, F. Cao, R. Torah, K. Yang, S. Beeby, J. Tudor, IEEE Sensors Journal 2014, 14, 393.

[7] S. Crea, M. Nann, E. Trigili, F. Cordella, A. Baldoni, F. J. Badesa, J. M. Catalán, L. Zollo, N. Vitiello, N. G. Aracil, S. R. Soekadar, Scientific Reports 2018, 8, 10823.

[8] M. Betke, J. Gips, P. Fleming, IEEE Transactions on Neural Systems and Rehabilitation Engineering 2002, 10, 1.

[9] J. Kim, X. Huo, J. Minocha, J. Holbrook, A. Laumann, M. Ghovanloo, IEEE Transactions on Biomedical Engineering 2012, 59, 1787.

[10] X. Huo, J. Wang, M. Ghovanloo, IEEE transactions on neural systems and rehabilitation engineering 2008, 16, 497.

[11] T. Le, First Aid for the Basic Sciences: Organ Systems, McGraw-Hill Education, 2011.

[12] C. G. Pinheiro, E. L. Naves, P. Pino, E. Losson, A. O. Andrade, G. Bourhis, Biomedical engineering online 2011, 10, 31.

[13] E. R. Kandel, J. H. Schwartz, T. M. Jessell, D. o. Biochemistry, M. B. T. Jessell, S. Siegelbaum, A. Hudspeth, Principles of neural science, Vol. 4, McGraw-hill New York, 2000.

[14] R. Hardie, A. Lees, Journal of Neurology, Neurosurgery \& Psychiatry 1988, 51, 850.

[15] A. S. Almansouri, N. A. Alsharif, M. A. Khan, L. Swanepoel, A. Kaidarova, K. N. Salama, J. Kosel, Advanced Materials Technologies 2019, 4, 1900493.

[16] A. Miyamoto, S. Lee, N. F. Cooray, S. Lee, M. Mori, N. Matsuhisa, H. Jin, L. Yoda, T. Yokota, A. Itoh, Nature nanotechnology 2017, 12, 907.

[17] N. A. Taylor, C. A. Machado-Moreira, Extreme physiology \& medicine 2013, 2, 4.

[18] W. Wang, Z. Yao, J. C. Chen, J. Fang, Journal of Micromechanics and Microengineering 2004, 14, 1321.

[19] M. A. Khan, H. Mohammed, J. Kosel, Advanced Engineering Materials 2018, 20, 1800492.

[20] Y. Chen, B. Lu, Y. Chen, X. Feng, Scientific reports 2015, 5, 11505.

[21] Intel, Vol. 2020, Intel, 2018.

[22] S. H. Ralston, I. D. Penman, M. W. Strachan, R. Hobson, Davidson's Principles and Practice of Medicine E-Book, Elsevier Health Sciences, 2018.

[23] Tecla, Vol. 2020, 2019.

[24] G. A. Device, Vol. 2020.

[25] Tobii, Vol. 2020, Tobii dunavox, 2020. 


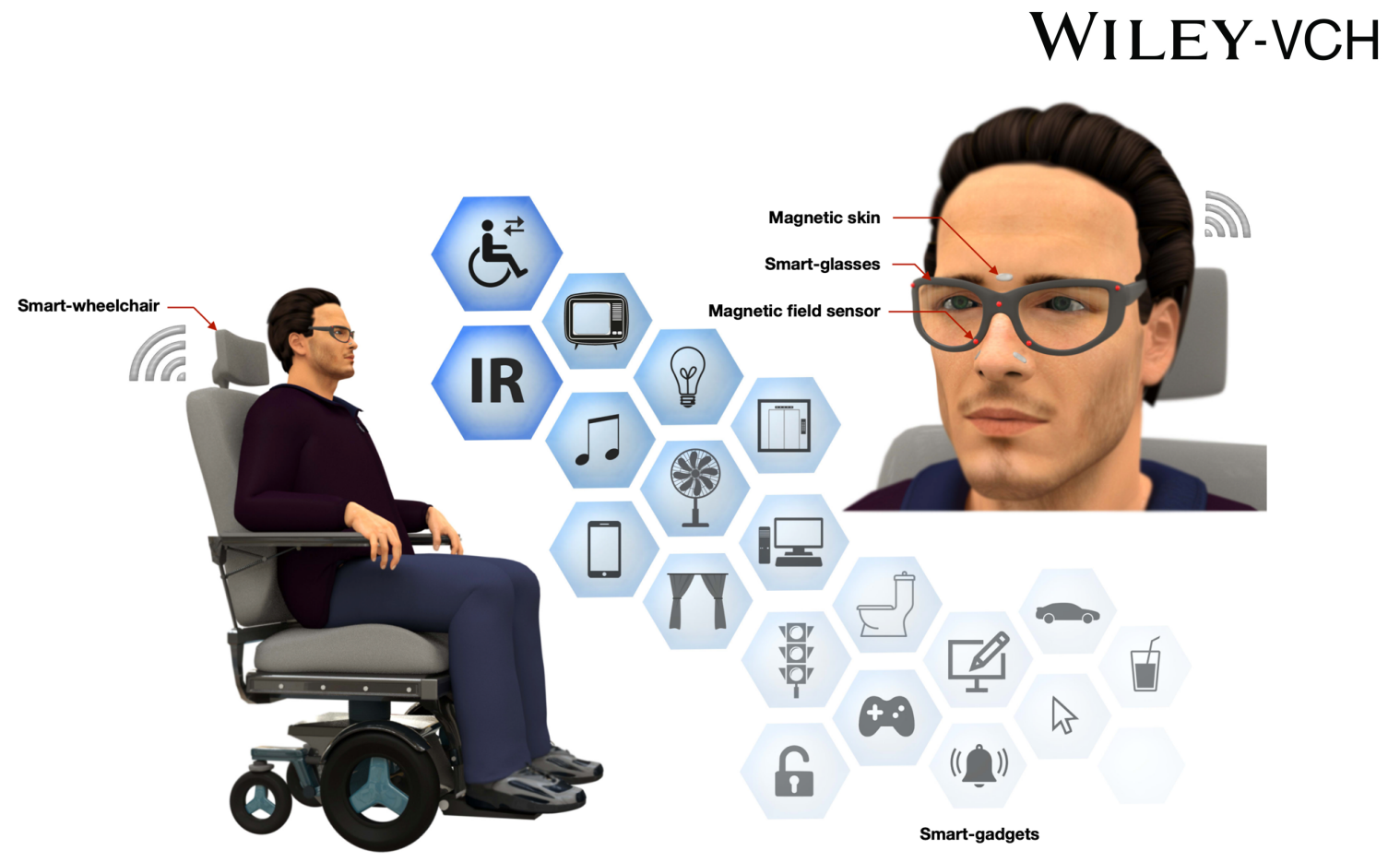

Figure 1. Assistive magnetic skin system (AM2S) for quadriplegics. It allows them to move around and to control their environment (e.g., television, lights, curtains, etc.) independently, using their facial expressions. The system consists of 1) magnetic skins attached to different places on the face; 2) smart-glasses with integrated magnetic field sensors, to track the magnetic skins, thus the facial expressions of the user, and built-in Bluetooth module; 3) a smart-wheelchair with builtin Bluetooth module and infrared (IR) module; and 4) smart-gadgets with IR communication. Note, the smart-glasses communicates with the smart-wheelchair using the Bluetooth link. The smart wheelchair communicates with the smart-gadgets using a line-of-sight IR link. Such a configuration allows for the directive control of the smart-gadget facing the user. 


\section{WILEY-VCH}
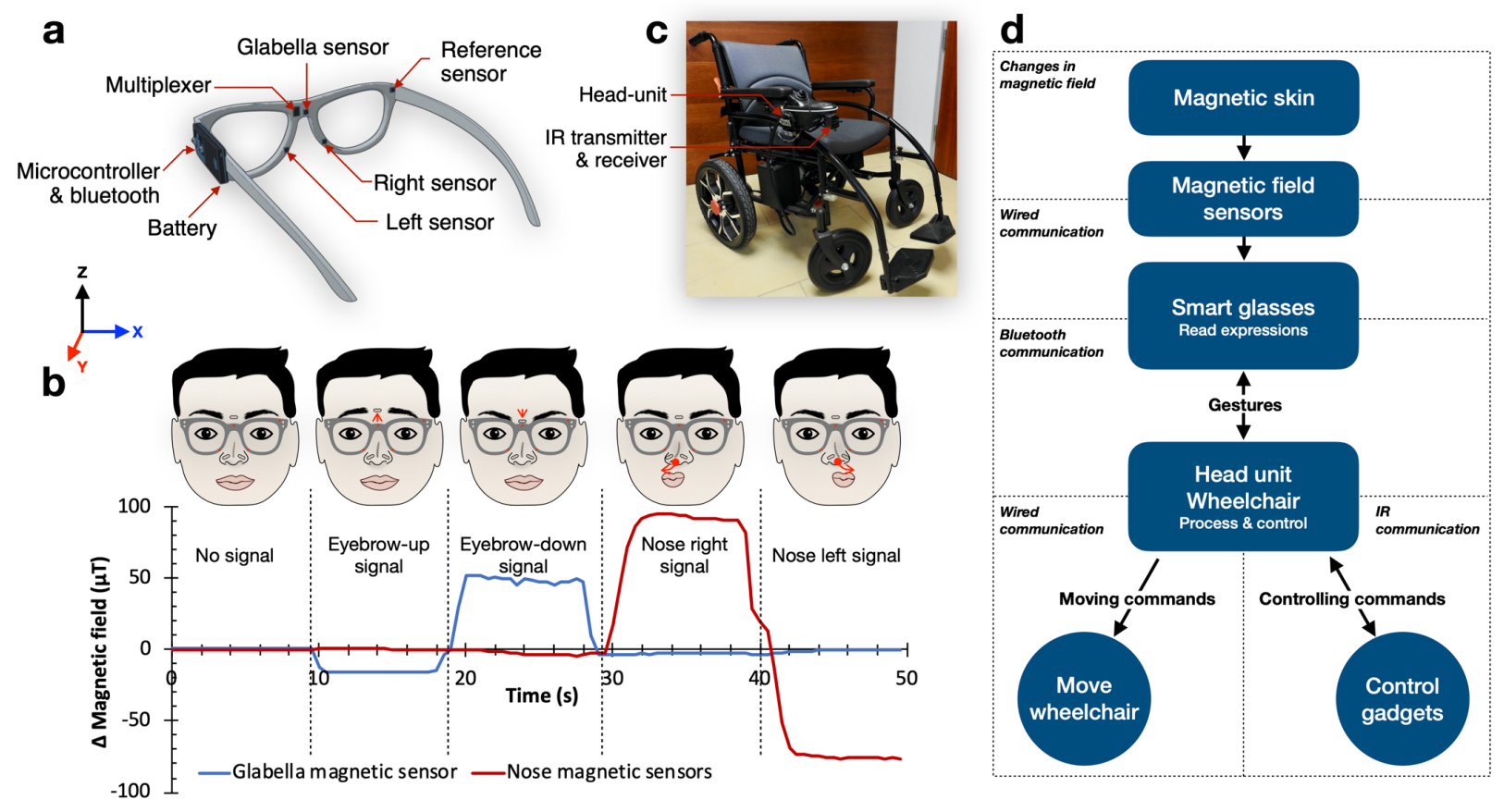

Figure 2. Implementation of the AM2S. a) It consists of smart-glasses with built-in magnetic field sensors (one acting as a reference, and three located near the magnetic skins), a microcontroller, a battery and a Bluetooth module for wireless communication. b) The smart-glasses detect the facial expressions. When there is no expression, there are no changes in the magnetic field signals. Eyebrows-up and eyebrows-down expressions result in changing the distance between the Glabella sensor and the magnetic skin, thus, respectively lowering and increasing the magnetic-filed measured by the glabella sensor. Similarly, turning the nose right or left results in increasing and decreasing the magnetic signals measured by the nose sensors. Note, all measurements are referenced to the reference sensor for cancelation of external magnetic noise like the Earth's magnetic field. Also, the nose measurements are differential for better signal-to-noise ratio. c) A photograph of the smart-wheelchair showing the head-unit that consists of a microcontroller, a Bluetooth module, and an IR communication module. d) Flow chart of the AM2S. The facial expressions are translated into changes in the magnetic field. These changes are detected using the magnetic field sensors within the smart-glasses and converted into electrical signals. The microcontroller translates these electrical signals into commands and sends them to the head-unit using Bluetooth communication. The head-unit further process the commands and sends them to the wheelchair (using wired communication) to move the wheelchair or to the IR module to control the smart-gadget in front of the wheelchair using line-of-sight IR communication. 


\section{WILEY-VCH}

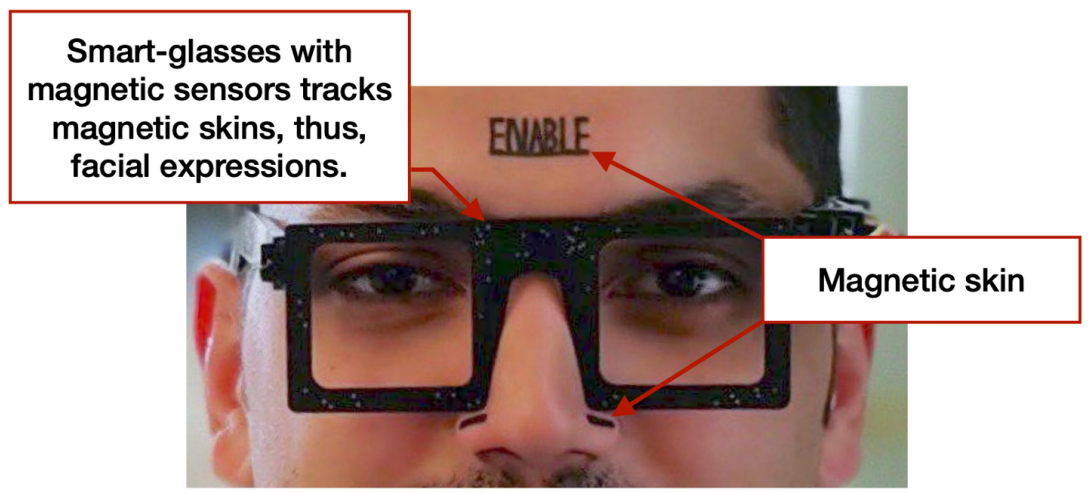

Figure 3. A photograph of a user wearing the smart-glasses and magnetic skins with different shapes and attached to different locations on the face. 


\section{WILEY-VCH}
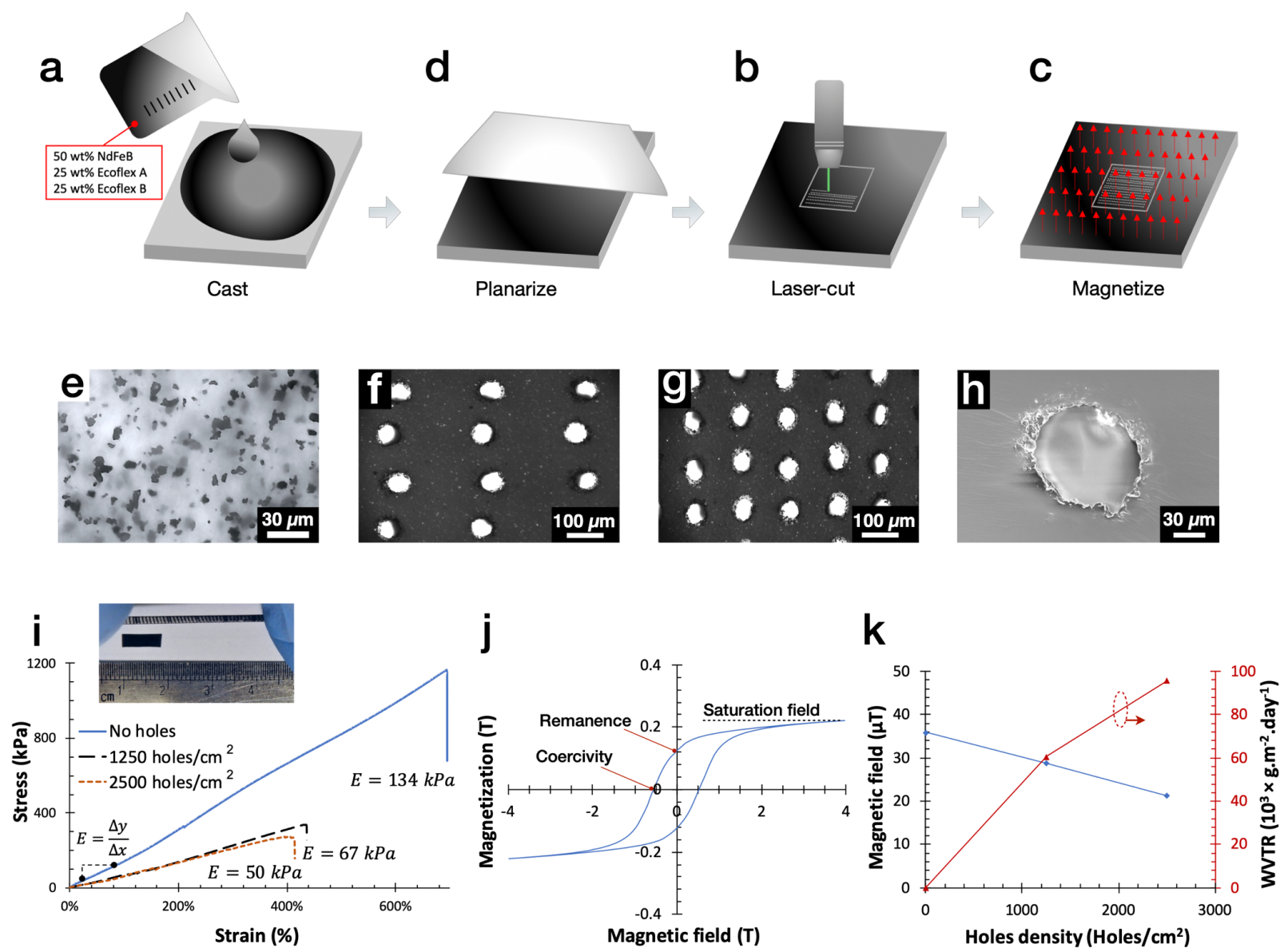

Figure 4. The breathable magnetic skin is fabricated by a) mixing EcoFlex with the magnetic powder, then casting it on a flat substrate; b) planarizing the mixture into the desired thickness (i.e., $120 \mu \mathrm{m}$ in this case); c) machining micro-holes using a ytterbium fiber laser after the mixture is dry; and d) finally, magnetizing it in the desired direction. e) A photograph of a diluted magnetic skin (i.e., 1:10 wt\% magnetic powder to elastomer) showing a uniform distribution of the magnetic microparticles. f-g) Photographs of the breathable magnetic skin with 1250 holes $/ \mathrm{cm}^{2}$ and 2500 holes $/ \mathrm{cm}^{2}$, respectively. h) An SEM image of the breathable magnetic skin showing the morphology of a hole. i-k) Characterization of a magnetic skin with a 1:1 wt\% of EcoFlex to magnetic powder. i) Stress-strain curves with Young's modulus of $134 \mathrm{kPa}, 67 \mathrm{kPa}$ and $50 \mathrm{kPa}$ for no-holes, 1250 holes $/ \mathrm{cm}^{2}$ and 2500 holes $/ \mathrm{cm}^{2}$ magnetic skin samples, respectively. The inset shows a magnetic skin sample with 1250 holes $/ \mathrm{cm}^{2}$ stretched to more than $300 \%$ elongation without breaking. j) Magnetization curve of the magnetic skin showing a coercivity of about 0.56 $\mathrm{T}$ and a remanent magnetization of $126 \mathrm{mT}$. k) The effect of micro-holes on the breathability and magnetization of the magnetic skin. 


\section{WILEY-VCH}

Table 1. Training time and success rate of using the AM2S system

\begin{tabular}{ccccccc}
\hline \hline & \multicolumn{3}{c}{ Success rate } & Total accidental & $\begin{array}{c}\text { Training time } \\
\text { (min.) }\end{array}$ \\
\cline { 2 - 7 } & Eyebrows-up & Eyebrows-down & Nose-right & Nose-left & 10 \\
\hline \hline User 1 & $10 / 10$ & $10 / 10$ & $10 / 10$ & $10 / 10$ & 0 & 12 \\
User 2 & $10 / 10$ & $8 / 10$ & $10 / 10$ & $10 / 10$ & 1 & 13 \\
User 3 & $10 / 10$ & $10 / 10$ & $10 / 10$ & $10 / 10$ & 0 & 11.6 \\
\hline Average & $100 \%$ & $93 \%$ & $100 \%$ & $100 \%$ & 0.3 & \\
\hline \hline
\end{tabular}

* The total number of accidental activations of the AM2S during performing the success rate test or during a two-minutes session of free talk. During the free talk, the user is allowed to move freely and to laugh. 


\section{WILEY-VCH}

Table 2. Assistive technologies for quadriplegics

\begin{tabular}{|c|c|c|c|}
\hline System & Description & $\begin{array}{c}\text { Supports severe } \mathbf{C 1} \text { and } \mathrm{C2} \\
\text { quadriplegics }\end{array}$ & Advantages / limitations \\
\hline Proposed AM2S & $\begin{array}{l}\text { Imperceptible magnetic skin attached } \\
\text { to different locations on the face and } \\
\text { tracked by magnetic field sensors } \\
\text { integrated into a smart-glasses. }\end{array}$ & $\begin{array}{l}\text { Yes, and highly customizable } \\
\text { to suit individual's need. }\end{array}$ & $\begin{array}{l}\text { Supports many action commands, customizable } \\
\text { to the user's need, and compatible with severe } \\
\text { quadriplegics. } \\
\text { - Requires wearing a smart-glasses. }\end{array}$ \\
\hline $\begin{array}{l}\text { Camera-based } \\
\text { systems }^{\mid 21]}\end{array}$ & $\begin{array}{l}\text { A 3D camera mounted in front of the } \\
\text { user (i.e., attached to a wheelchair) } \\
\text { and used to track the facial expression. }\end{array}$ & $\begin{array}{l}\text { No, severely injured people } \\
\text { might have breathing problems } \\
\text { and requires apparatus attached } \\
\text { for breathing. }{ }^{[1,22]}\end{array}$ & $\begin{array}{l}\text { Dose not require the physical attachment of any } \\
\text { device on the body. } \\
\text { - Requires extensive computational and electrical } \\
\text { power. There is a delay between the gesture and } \\
\text { the action. Requires continuous attention of the } \\
\text { user. }\end{array}$ \\
\hline $\begin{array}{l}\text { Voice } \\
\text { assistant }^{[23]}\end{array}$ & $\begin{array}{l}\text { A nearby microphone used for voice } \\
\text { and commands recognition. }\end{array}$ & $\begin{array}{l}\text { No, severely injured people can } \\
\text { have speech problems. }{ }^{[11,22]}\end{array}$ & $\begin{array}{l}\text { The system can be hidden in the surrounding, } \\
\text { and works well indoor if the user can talk clearly. } \\
\text { - There is a delay between the gesture and the } \\
\text { action. Voice recognition does not work in a } \\
\text { noisy environment. Subjective to accidental } \\
\text { activation. }\end{array}$ \\
\hline $\begin{array}{l}\text { Tongue assistive } \\
\text { system }^{[9]}\end{array}$ & $\begin{array}{l}\text { A magnet attached to the tongue and } \\
\text { tracked using sensors placed in } \\
\text { proximity to the mouth. }\end{array}$ & $\begin{array}{l}\text { Yes, as long as the users can } \\
\text { freely control their tongue. }\end{array}$ & $\begin{array}{l}\text { - Compatible with severe quadriplegics } \\
\text { - Invasive and the user cannot talk while using the } \\
\text { system. }\end{array}$ \\
\hline $\begin{array}{l}\text { Joysticks and } \\
\text { head trackers }^{[24]}\end{array}$ & $\begin{array}{l}\text { Electronics placed near the face or } \\
\text { integrated into eyeglasses to track the } \\
\text { movement of the head. }\end{array}$ & $\begin{array}{l}\text { No, severely injured people } \\
\text { have very limited head and } \\
\text { neck movement. }\end{array}$ & $\begin{array}{l}\text { - Simple and easy to use. } \\
\text { Limited gestures that are not enough to control } \\
\text { the surroundings. Not compatible with severe } \\
\text { injuries. }\end{array}$ \\
\hline $\begin{array}{l}\text { Gaze and eye- } \\
\text { tracking }\end{array}$ & $\begin{array}{l}\text { Infrared light (or other methods) } \\
\text { tracks eye-movements. }\end{array}$ & $\begin{array}{l}\text { Yes, as long there is no } \\
\text { obstacle in the way between the } \\
\text { eyes and the detector. }\end{array}$ & $\begin{array}{l}\text { Dose not require to physically attach any device } \\
\text { directly on the body. } \\
\text { - Requires continuous attention and offers limited } \\
\text { action commands. }\end{array}$ \\
\hline
\end{tabular}




\section{WILEY-VCH}

A novel and comprehensive assistive magnetic skin system for severely injured quadriplegics (i.e., $\mathrm{C} 1$ and $\mathrm{C} 2$ injuries) is presented. It is based on wearable and breathable magnetic tattoos, smartglasses, a smart-wheelchair, and smart-gadgets. The system is demonstrated for moving a wheelchair, and controlling the surroundings for indoor and outdoor applications (e.g., lights, curtain, doors, air-condition, elevators, pedestrian lights, etc.).

A. S. Almansouri ${ }^{*}$, L. Upadhyaya, S. P. Nunes, K. N. Salama, and J. Kosel ${ }^{*}$

An Assistive Magnetic Skin System: Enabling Technology for Quadriplegics

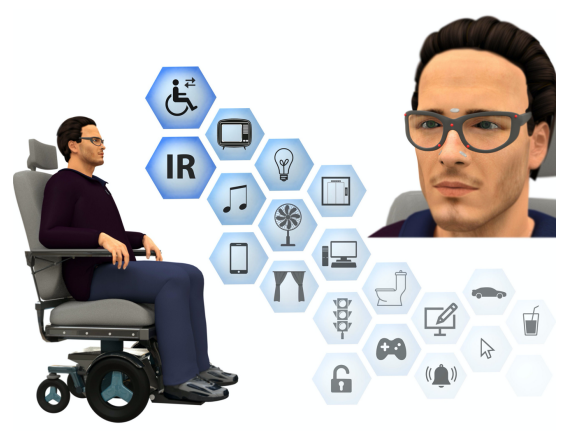




\section{WILEY-VCH}

Copyright WILEY-VCH Verlag GmbH \& Co. KGaA, 69469 Weinheim, Germany, 2018.

Supporting Information

An Assistive Magnetic Skin System: Enabling Technology for Quadriplegics

Abdullah S. Almansouri ${ }^{*}$, Lakshmeesha Upadhyaya, Suzana P. Nunes, Khaled N. Salama, and Jurgen Kosel 


\section{WILEY-VCH}

\section{Supplementary Information 1}

Figure S1a shows the block diagram of the smart-glasses. It consists of a microcontroller with a built-in Bluetooth 4.0 module (Bluno nano, DFRobot), 8-channels multiplexer (PCA9548), multi-axis magnetic field sensors (BM1422AGMV), and a $5 \mathrm{~V}$ battery. To enhance the signal-tonoise ratio, the magnetic field at the nose is measured differentially (i.e., $H_{\text {nose }}=H_{\text {nose }}$ right $-H_{\text {nose }}$ left, where $H$ is the magnetic field measured by a magnetic field sensor). Furthermore, a reference magnetic field sensor is used for offset cancelation of any external magnetic field (e.g., Earth's magnetic field). Figure S1 b and c show photographs of the PCB of the smart-glasses which is shaped as a frame of eyeglasses. 
a

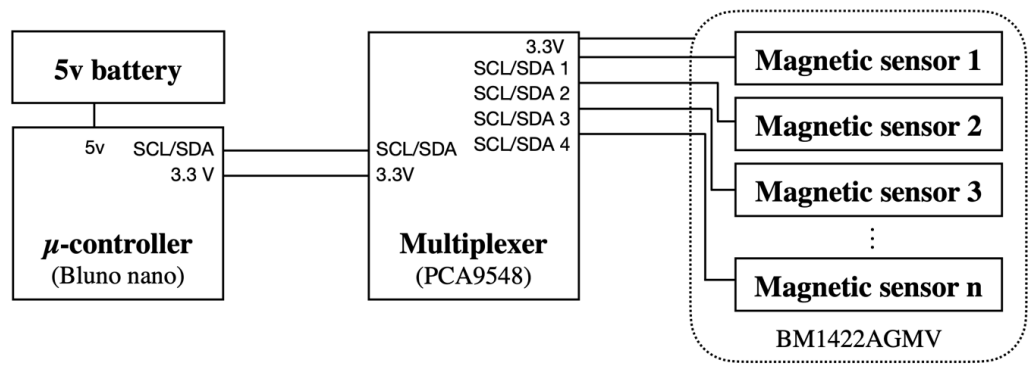

b
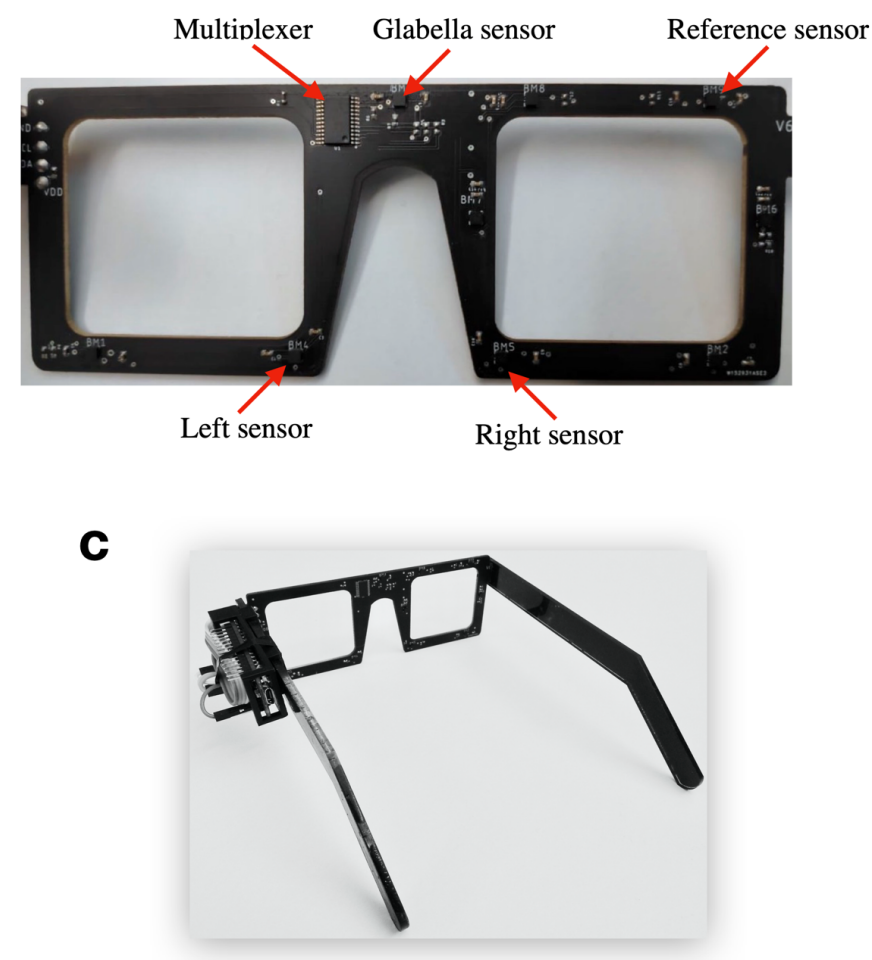

Figure S1. a) A block diagram of the smart-glasses that consist of a micro-controller, a multiplexer, and a number of magnetic field sensors, which is 4 in this case. One sensor is used as a reference sensor for offset cancelation, and the rest of the sensors are used to measure the nearby magnetic skins. $b$ and c) photographs of the smart-glasses with a PCB shaped as the frame of eyeglasses. 


\section{WILEY-VCH}

\section{Supplementary Information 2}

Table S1 shows the list of gestures that the AM2S currently supports. Furthermore, the digital codes to activate these gestures are also presented. These codes are representations for the sequence of movements within a given time to activate a certain gesture. Note that, more gestures can be added as needed simply by defining additional code sequence. Figure S2 shows the normalized analog signals detected by the smart glasses and the corresponding digital code. Below a certain threshold value, the signal is determined as "no-expression" (i.e., $\mathrm{N}_{0}$ representing no nose-movement and $\mathrm{B}_{0}$ for no eyebrows-movement). Once the analog magnetic field passes the threshold value, a digital code is generated (i.e., $\mathrm{B}_{1}$ for eyebrow-down, $\mathrm{B}_{-1}$ for eyebrow-up, $\mathrm{N}_{1}$ for nose-right, and $\mathrm{N}_{-1}$ for nose-left movements).

Table S1. List of gestures supported by the AM2S

\begin{tabular}{l|l|l}
\hline \multicolumn{1}{c|}{ Gestures } & \multicolumn{1}{c}{$\begin{array}{c}\text { Digital } \\
\text { representation }\end{array}$} & \multicolumn{1}{c}{ Application } \\
\hline \hline 1- No-expressions & $\mathrm{B}_{0} \& \mathrm{~N}_{0}$ & A null command where the user would like to stand-still. \\
3- Eyebrow-down & $\mathrm{B}_{1} \& \mathrm{~N}_{0}$ & Move the wheelchair forward or turns gadgets ON. \\
4- Nose-right & $\mathrm{B}_{0} \& \mathrm{~N}_{1}$ & $\begin{array}{l}\text { Turn the wheelchair right or, for example, move the cursor } \\
\text { of a computer right. }\end{array}$ \\
5- Nose-left & $\mathrm{B}_{0} \& \mathrm{~N}_{-1}$ & Turn the wheelchair left or, for example, move the cursor of \\
& a computer left.
\end{tabular}




\section{WILEY-VCH}

\begin{tabular}{|c|c|c|}
\hline $\begin{array}{l}\text { 6- Eyebrow-up } \\
\text { and nose-right }\end{array}$ & $\mathrm{B}_{-1} \& \mathrm{~N}_{1}$ & $\begin{array}{l}\text { Turn the wheelchair right while still moving forward or, for } \\
\text { example, move the cursor of a computer diagonally (i.e., } \\
\text { up-right). }\end{array}$ \\
\hline $\begin{array}{l}\text { 7- Eyebrow-up } \\
\text { and nose left }\end{array}$ & $\mathrm{B}_{-1} \& \mathrm{~N}-1$ & $\begin{array}{l}\text { Turn the wheelchair left while still moving forward or, for } \\
\text { example, move the cursor of a computer diagonally (i.e., } \\
\text { up-left). }\end{array}$ \\
\hline $\begin{array}{l}\text { 8- Eyebrows- } \\
\text { down and nose- } \\
\text { right }\end{array}$ & $\mathrm{B}_{1} \& \mathrm{~N}_{1}$ & $\begin{array}{l}\text { Turn the wheelchair right while still moving backward or, } \\
\text { for example, move the cursor of a computer diagonally (i.e., } \\
\text { down-right). }\end{array}$ \\
\hline $\begin{array}{l}\text { 9- Eyebrows- } \\
\text { down and nose- } \\
\text { left }\end{array}$ & $\mathrm{B}_{1} \& \mathrm{~N}_{-1}$ & $\begin{array}{l}\text { Turning the wheelchair left while still moving backward or, } \\
\text { for example, move the cursor of a computer diagonally (i.e., } \\
\text { down-left). }\end{array}$ \\
\hline $\begin{array}{l}\text { 10- Double } \\
\text { eyebrow-up }\end{array}$ & $\begin{array}{l}{\left[\mathrm{B}_{0} \rightarrow \mathrm{B}_{-1} \rightarrow\left(\mathrm{B}_{0}\right.\right.} \\
\left.\| \mathrm{B}_{1}\right) \rightarrow \mathrm{B}_{-1} \rightarrow \\
\left.\mathrm{B}_{0}\right] \& \mathrm{~N}_{0}\end{array}$ & $\begin{array}{l}\text { Select option, for example, for gadgets with multiple } \\
\text { options. }\end{array}$ \\
\hline $\begin{array}{l}\text { 11- Triple } \\
\text { eyebrow-up }\end{array}$ & $\begin{array}{l}{\left[\mathrm{B}_{0} \rightarrow \mathrm{B}_{-1} \rightarrow\left(\mathrm{B}_{0}\right.\right.} \\
\left.\| \mathrm{B}_{1}\right) \rightarrow \mathrm{B}_{-1} \rightarrow \\
\left(\mathrm{B}_{0} \| \mathrm{B}_{1}\right) \rightarrow \mathrm{B}_{-1} \\
\left.\rightarrow \mathrm{B}_{0}\right] \& \mathrm{~N}_{0}\end{array}$ & $\begin{array}{l}\text { Enter a controlling mode or back to the wheelchair mode. } \\
\text { For example, when triple eyebrow-up is detected, the } \\
\text { wheelchair stops (once a smart gadget is detected), and } \\
\text { control switches to the smart gadgets in front of it. }\end{array}$ \\
\hline
\end{tabular}




\begin{tabular}{l|l|l}
$\begin{array}{l}\text { 12- Double } \\
\text { eyebrow-up and }\end{array}$ & $\begin{array}{l}\left(\mathrm{B}_{0} \rightarrow \mathrm{B}_{-1} \rightarrow\left(\mathrm{B}_{0}\right.\right. \\
\left.\| \mathrm{B}_{1}\right) \rightarrow \mathrm{B}_{-1} \rightarrow\end{array}$ & For example, a right-click on a computer. \\
nose-right & $\left.\mathrm{B}_{0}\right) \& \mathrm{~N}_{1}$ \\
13- Double & $\left(\mathrm{B}_{0} \rightarrow \mathrm{B}_{-1} \rightarrow\left(\mathrm{B}_{0}\right.\right.$ & For example, a left-click on a computer. \\
eyebrow-up and & $\left.\| \mathrm{B}_{1}\right) \rightarrow \mathrm{B}_{-1} \rightarrow$ & \\
nose-left & $\left.\mathrm{B}_{0}\right) \& \mathrm{~N}_{-1}$ & \\
\hline \hline
\end{tabular}

$\overline{{ }^{a} \text { Digital representation of eyebrows and nose movements to activate a specific gesture. The digital codes are }}$ highlighted in Figure S2.

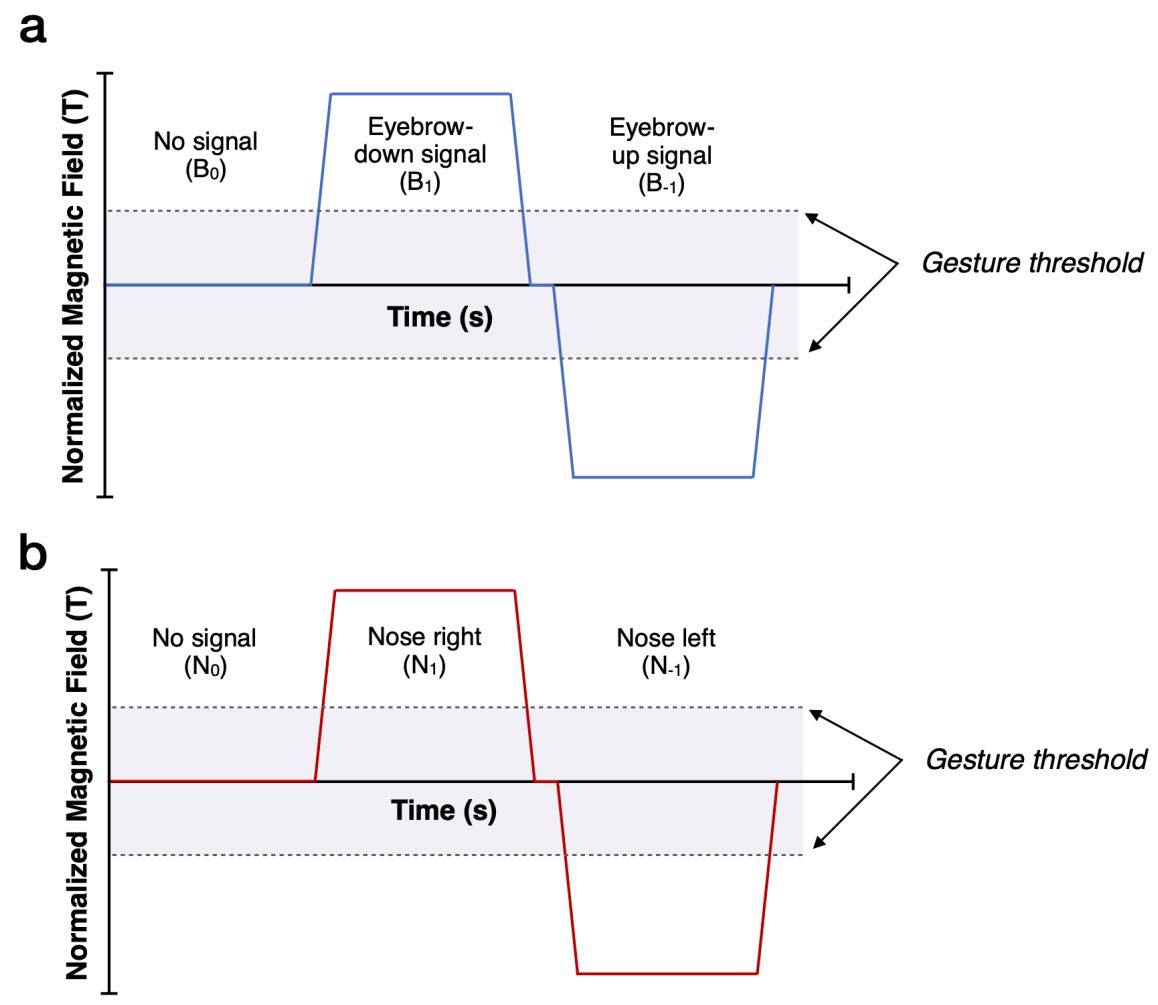

Figure S2. Digital representation of the facial expressions detected by the smart-glasses. Once the magnetic field detected by the smart-glasses passes a certain threshold value, the smart-glasses converts the signal into a digital value. a) For the case of the Glabella magnetic field sensor, the 


\section{WILEY-VCH}

digital signal is $\mathrm{B}_{0}$ when the eyebrow is in normal position, $\mathrm{B}_{1}$ when the eyebrow is down, and $\mathrm{B}$. 1 when the eyebrow is up. b) For the case of the Nose magnetic field sensor, the digital signal is $\mathrm{N}_{0}$ when the nose is in a normal position, $\mathrm{N}_{1}$ when the nose is tilted to the right, and $\mathrm{N}_{-1}$ when the nose is tilted to the left. 


\section{WILEY-VCH}

\section{Supplementary Information 3}

The block diagram of the head-unit is presented in Figure S3. It consists of a microcontroller with a built-in Bluetooth 4.0 module (Bluno nano, DFRobot), two Digital-toAnalog converters (DACs, MCP4725), an IR transmitter and IR receiver operating at $38 \mathrm{kHz}$. The DACs are connected to the joystick-input in the wheelchair and supplies a voltage between 0 to 5 $\mathrm{V}$ to move the wheelchair in different directions. For example, $0 \mathrm{~V}$ output from DAC1 moves the wheelchair in the reverse direction, while $5 \mathrm{~V}$ move the wheelchair forward. Similarly, $0 \mathrm{~V}$ output from DAC2 turns the wheelchair right, and $5 \mathrm{~V}$ turns the wheelchair left. Note, the head-unit, the IR transmitter, and the IR receiver are powered by the wheelchair.

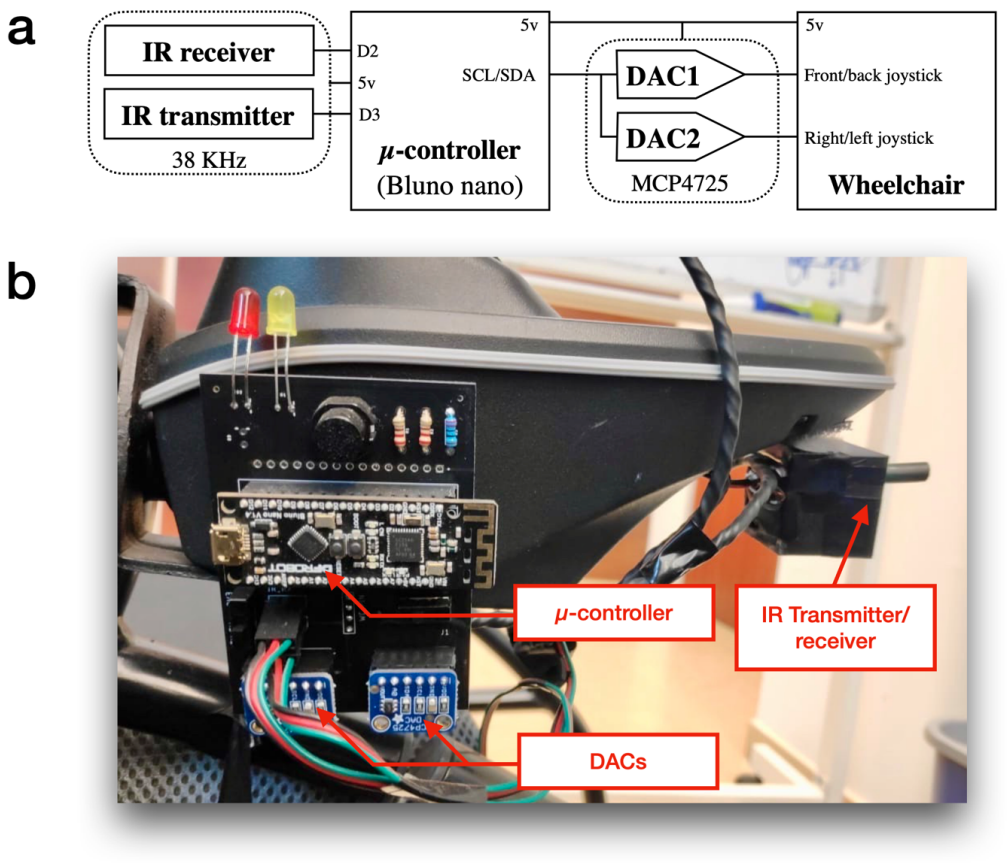

Figure S3. a) Block diagram for the head-unit of the wheelchair. It consists of a microcontroller with integrated Bluetooth module, two Digital-to-Analog converters, an IR transmitter and an IR receiver operating at $38 \mathrm{kHz}$. The Analog-to-Digital converters are interfaced with the joystickinput of the wheelchair for controlling the wheelchair. b) Photograph of the actual PCB integrated to the wheelchair. 


\section{WILEY-VCH}

Figure S4 shows the block diagram of the smart gadget. It consists of a microcontroller (i.e., Bluno nano, DFRobot), an IR transmitter and IR receiver operating at $38 \mathrm{kHz}$, and an interface with the gadgets. The interface might be different depending on the gadget. For example, if the gadget is a computer, the interface can simply be a USB cable. However, if the gadget is a lightswitch, the interface would be a high-power relay or a DAC.

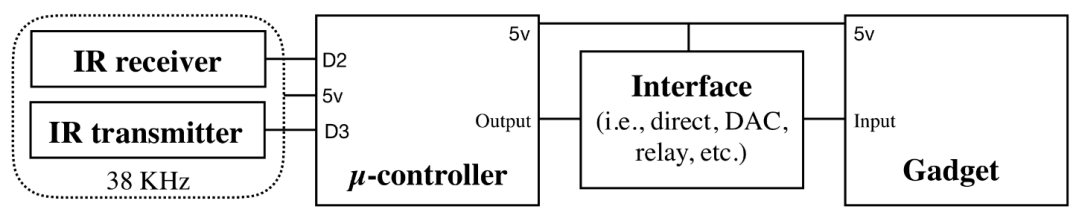

Figure S4. Block diagram of a smart-gadget. It consists of a microcontroller, an IR transmitter, an IR receiver, and an interface with the gadget. The interface can be different from one gadget to another, and it depends on the nature of the gadget. 


\section{WILEY-VCH}

\section{Supplementary Information 4}

The combination of the smart-glasses, the smart-wheelchair, and the smart-gadgets allows the users to move around and to control their environments. The smart-glasses track the movement of the magnetic skin, once the movement passes a certain threshold, the corresponding gesture is triggered. Note that the threshold value is defined as $70 \%$ of the maximum/minimum magnetic field corresponding to each of the eyebrows-up, eyebrows-down, nose-right, and nose-left gestures for a certain user. This $70 \%$ value was found experimentally to mitigate the accidental activations by talking, laughing, etc. for which we found values of less than $60 \%$ Nevertheless, this value can be custom-tuned to suits an individual's needs. Once a gesture is detected, it is then transmitted to the head-unit using Bluetooth communication. The head-unit then determines the type of the action commands (i.e., moving commands or controlling commands). For the case of the moving commands, the head-unit sends the commands to the wheelchair to move according to the gestures (i.e., the facial expression). As a consequence, the chair moves forward, moves backward, turns right, or turns left.

For the case of the controlling commands, the head-unit sends the commands to the IR link to control the smart-gadgets within the line-of-sight. These commands are further processed at the smart-gadgets for the intended use, which can be different from one smart-gadget to another. For example, an eyebrow-up command can turn on the light, and the same gesture can be used to move the cursor up on a computer.

The triple eyebrow-up command is used as a search/disable command, and it is reserved for switching between the moving commands and the controlling commands. In other words, if a triple eyebrow-up gesture is triggered while the user is at the moving commands, the head-unit starts searching for a smart-gadget (using the IR link) within the line-of-sight, yet, the wheelchair 


\section{WILEY-VCH}

can move normally using the moving commands. Once a smart gadget is detected, the moving commands are terminated, and the head-unit starts controlling the smart gadget using the controlling commands. For example, if the user wants to turn on the lights, the user starts by triggering the triple eyebrow-up gesture and points the wheelchair (i.e., using the eyebrowup/down and nose- right/left gestures) to the light-switch. Once the light-switch is within reach, the wheelchair stops moving, and the user is now connected to the light-switch. On the other hand, if a triple eyebrow-up gesture is triggered while the user is at the controlling commands (e.g., connected to the light-switch), the head-unit basically switches back to the moving commands. 


\section{WILEY-VCH}

\section{Supplementary Information 5}

Figure S5 shows the impact of having different concentrations of magnetic powder on the properties of the magnetic skin. Increasing the concentration of the magnetic powder results in stiffer material with higher remanent magnetization, and vice versa. Thus a $1: 1 \mathrm{wt} \%$ magnetic skin is the optimal choice that maintains a high magnetic field and high flexibility. This enhanced performance is maintained over more than 1000 stress cycles (i.e., stretching and relaxing) with no evidence of significant changes in the physical properties.

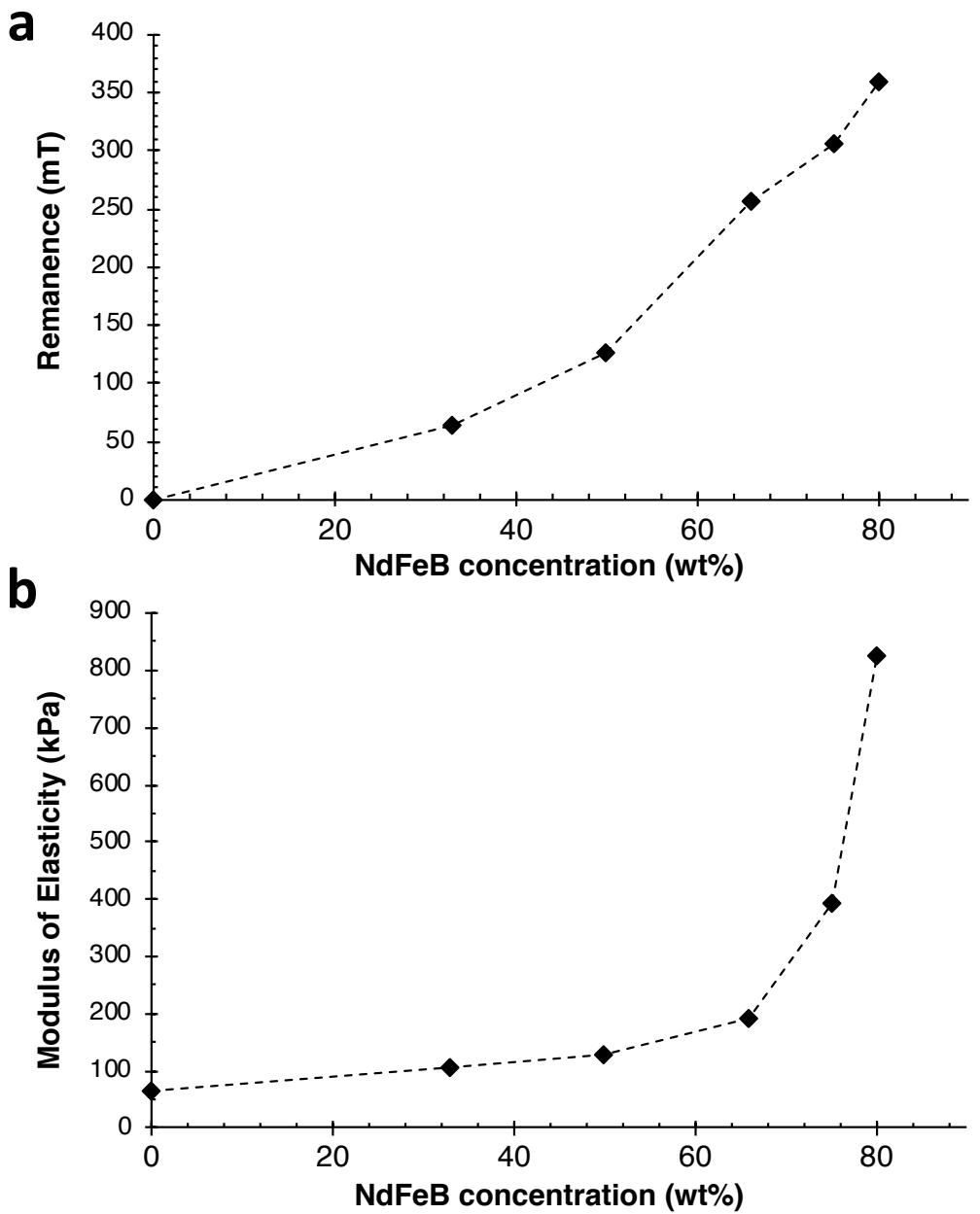

Figure S5. The impact magnetic powder $(\mathrm{NdFeB})$ concentration on a) the remanence and $b$ ) the modulus of elasticity for the magnetic skin. 